\title{
JEJ buys biotech with promising prostate cancer therapy
}

Johnson \& Johnson has bought Cougar Biotechnology, which has a pipeline focused on oncology, for almost US\$1 billion. The company's lead compound is abiraterone, an inhibitor of cytochrome P450 17 (CYP17) - an enzyme involved in androgen and oestrogen biosynthesis - which has recently shown positive Phase II results in castrationresistant prostate cancer (CRPC).

As Jan-Erik Damber, Professor and Chairman in the Department of Urology at the University of Gothenburg, Sweden, explains: "Mortality from prostate cancer is fully attributed to the disseminated form castration-resistant prostate cancer. At this stage of the cancer, there are at present almost no effective drugs. The only exception is Taxotere chemotherapy, which has only a small (but significant) survival benefit. Thus, there is an urgent need for new treatment options for this group of patients."

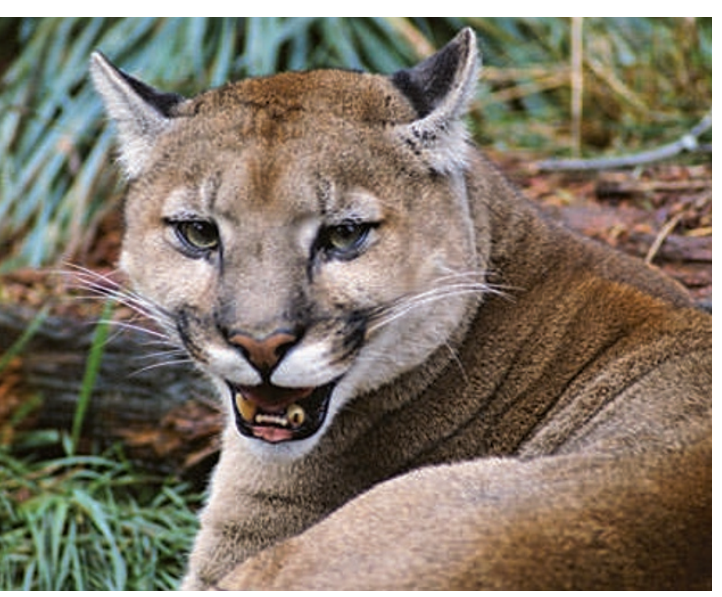

The Phase II trial of abiraterone included 42 patients with chemotherapy-naive CRPC that had experienced disease progression despite multiple treatments (J. Clin. Oncol. 26 May 2009; doi: 10.1200/JCO.2008.20.0642). Treatment with abiraterone reduced serum levels of prostate-specific antigen (PSA; a biomarker of prostate cancer) by more than $50 \%$ in 28 individuals and by more than $90 \%$ in 8 patients. Moreover, partial radiological responses (an indicator of tumour shrinkage) occurred in 9 out of 24 patients with measurable disease, and declines in circulating tumour cell counts were observed.

The beneficial effects of the drug could be due to its specific mechanism of interaction with prostate cancer cells, according to Damber: "CYP17 and androgen biosynthesis is upregulated in prostate cancer cells, thus providing these cells with androgen stimulation in an autocrine fashion. In addition, androgen receptor expression is frequently amplified and hypersensitive to androgenic signalling. These effects, together with the potential of inhibition of adrenal synthesis of androgens and precursors, could together form the rationale for treatment with a CYP17 inhibitor."

Moreover, clinical studies with abiraterone have increased the understanding of CRPC biology, notes Johann de Bono of the prostate cancer team at The Institute of Cancer Research, UK, and lead author of the Phase II study. "Although CRPC was for many years referred to as hormone-refractory prostate cancer, we have now renamed this disease castration-resistant prostate cancer; recent studies have confirmed that, in about $60-70 \%$ of men with CRPC, steroid hormones may continue to drive the expression of oncogenic ETS gene rearrangements. We have also recently published data suggesting that patients with the $E R G$ rearrangement are those most likely to benefit from abiraterone."

After many years without major treatment advances for prostate cancer, there are now several other investigational therapeutics that are raising new hopes. "Recent clinical data suggest that in addition to hormonal therapy or chemotherapy, immunotherapy is showing promise in prostate cancer," says James Gulley, Director of the Clinical Trials Group, Laboratory of Tumor Immunology and Biology, and Senior Clinician, Medical Oncology Branch at the National Cancer Institute, USA. "Randomized controlled double-blinded studies with the therapeutic vaccines Provenge (in Phase III) and Prostvac (in Phase II) suggest improved survival in men with metastatic chemotherapy-naive CRPC."

In addition, data presented at this year's American Society for Clinical Oncology (ASCO) meeting have shown that other mechanisms of modulating androgen activity might also be realistic. In Phase I-II trials, MDV3100 (ASCO abstract 5011) - a novel androgen receptor antagonist that retains antagonist properties in cells that overexpress androgen receptors (Science 324, 787-790; 2009) - showed positive effects in patients with CRPC, as assessed by measurements of PSA levels and circulating tumour cells, and tumour imaging. 